\title{
Heidegger: Ek-Sistence Authentique et
}

\section{Ethique comme Thanatologie}

La réponse à la question "Qui est l'être-là"? (Wer ist Dasein?), laquelle Heidegger pose dans Sein und Zeit (L'Etre et le temps), mène logiquement à la croyance au determinisme ou conditionnement socioculturel, et partant, à la décadence de l'identité personnelle ou à la perte du moi individuel. Selon la conception heideggerienne de l'inauthenticité, personne n'est soi-même; tout le monde est réduità un phénomène social pré-conscient connu sous le nom de "on" (das Man, en allemand). Une interprétation orthodoxe de la pensée heideggerienne insinue que ce que nous sommes vraiment dépend de ce Man anonyme. Nous devons vivre et agir comme "tout le monde", ou comme "n'importe qui", c'est-à-dire "les autres", "le on". Mais en étant et en agissant de cette manière, nous n'assumons aucune responsabilité personnelle pour nos actes.

Si mes opinions dépendent de ce que "l'on pense", ou mes reactions émotives dépendent de ce que "l'on éprouven, et mes actions, de la convention sociale du milieu dans lequel je vis, alors je pense, éprouve et agis comme membre du "on", ou mieux, pour utiliser l'expression de F. Copleston, comme membre d'une "collectivité impersonnelle plutôt que comme un individu précis". ${ }^{1}$ La collectivité impersonnelle de Copleston n'est rien d'autre que la masse qui, chez Kierkegaard, est la non-verité, puisqu'elle rend l'individu complètement impénitent et irresponsable, ou du moins elle affaiblit son sens de responsabilité en le réduisant à une fraction. ${ }^{2}$ Kierkegaard - à qui Heidegger doit un bon nombre de vocables existentialistes - excite à mettre fin à l'identité collective et aux rôles sociaux en faveur du respect pour l'individuel. De même, Heidegger invite Dasein à en finir avec sa capitulation à "personne" (Keiner) ou, ce qui revient au même, à "tout le monde". Cette

\footnotetext{
${ }^{1}$ Frederick Copleston. A History of Philosophy. New York: Doubleday, 1985. Vol. VIII, Chap. XVII, p. 340.

${ }^{2}$ S. Kierkegaard. The point of View. Traduit par W. Lowrie. London, 1939, p. 114. Cité par Copleston, op. cit., p. 340.
} 
invitation signifie que chacun doit s'assumer soi-même comme individu et recouvrer son soi authentique. Malheureusement, l'existence authentique à laquelle Heidegger invite consiste à se comprendre comme mourant et à accepter la mort. Elle n'a donc rien à voir ni avec l'être soi-même au sens existentiel-concret, ni avec l'agir libre et responsable. Pareillement, l'ethique dont il est question chez lui est aussi mystérieuse que sa conception d'existence. Cette éthique, dite "originelle", ne peut être comprise qu'a la lumière du concept d'existence. Exister authentiquement et éthiquement, c'est sortir de l'anonymat engendré par la dictature du Man pour mourir de sa propre mort. Une telle existence et l'éthique sont, ou semblent être, en dernière analyse, une thanatologie pure et simple, c'est-à-dire une thérie sur le phenomène de la mort aussi bien qu'une préparation psychologique à ce phénomène. Il s'agit non seulement de la mort en général, mais peut-être aussi de la mort du peuple juif sous le régime hitlérien.

\section{QUEST-CE QUE L'EXISTENCE (EK-SISTENCE?}

D'une façon générale, "existence" signifie le fait d'être. Le mot s'oppose d'une part à "essencen", comme le fait d'être à la nature de l'être; de l'autre à "néant", comme l'affirmation à la négation. En un sens fort, "existence" signifie réalité vivante ou réalité vécue, par opposition aux abstractions et aux théories. Les scolastiques opposent essentia et existentia: l'essence est la nature conceptuelle d'une chose; elle est conçue comme potentialité ou le pouvoir d'être; l'existence au contraire est la pleine actualite, ultima actualitas; elle apparaît ainsi comme s'ajoutant à l'essence. ${ }^{3}$ Dans Sein und Zeit, Heidegger écrit: "Das Wesen des Daseins liegt in seiner Existenz" ${ }^{4}$ c'est-à-dire, l'être de l'être-là réside dans son existence. Jean-Paul Sartre en a fait l'affirmation du principe

\footnotetext{
${ }^{3}$ A Lalande. Vocabulaire Technique et Critique de la Philosophhie. $12 \lambda$ €d. Paris: Presses Universitaires de France, 1976, p. 318.

${ }^{4}$ Martin Heidegger. Sein und Zeiz. (SZ). Tubingen: Max Niemyer Verlag, 1979, p. 42 Première moitie, in Jahrbuch fur Phanomenologie und phanomelogische Forschung. Halle, vol. 8 1927, 446 pages; G.A Bd.2. Traduction française: L'Etre et le Temps. Traduit de l'allemand par Rudolf Boem et Alphonse de Waelhens. Paris: Ed. Gallimard, 1964. Cette traduction est partielle). Dorénavant le sigle SZ sera utilise pour Sein und Zeit.
} 
premier de son existentialisme selon lequel, dans l'existant humain, "l'existence précède l'essence" Evidemment, ceci est une interprétation anthropologiquede la formule heideggerienne. Pour montrer que l'existence dont il est question chez lui n'a pas le moindre point commun avec la réalité de l'ego cogito, ni avec le principe de l'existentialisme sartrien, Heidegger adopte une nouvelle épellation: Eksistenz ou Ek-sistenz, croyant ainsi marquer le caractère extatique de cette structure fondamentale de l'être-homme.

L'eksistence n'est donc pas le fait d'exister; mais elle est au contraire, dans la perspective de "l'ontologie fondamentale", la présence extatique à l'Etre que l'homme est appelé à soutenir dans le destin de la vérité. Dans l'eksístence, l'homme assume le Dasein, c'est-à-dire le fait d'être: -LE-LA. Le "la", en quoi l'homme eksiste, est la zone d'éclaircie de l'Etre. Tel est le sens ultime de "l'existence" dont parle Heidegger dans Sein und Zeit. ${ }^{6}$ L'existence de l'être-là est bidimensionnelle: elle est de prime abord et le plus souvent inauthentique; ensuite elle est, ou elle peut devenir authentique.

\section{LTNAUTHENTICITE OU L'EXISTENCE INAUTHENTIQUE}

Heidegger croit que tout Dasein débute par l'existence inauthentique, parce que la décheance (das Verfallen) est un de ses existentiaux ou éléments constitutifs. De même, la tyrannie exercée par le 'on' (das Man) et le passage dans le monde de la préoccupation (in der besorgten Welt) causent l'inauthenticité ou la perte d'identité au Dasein. Le problème d'inauthenticité consiste donc à répondre à la question suivante: "Qui est celui qui assume l'être de la coexistence quotidienne"??

\section{A. Le 'On' (das Han)}

Le 'on' est le sujet, c'est-à-dire celui qui assume la coexistence

${ }^{5} J .-P$. Sartre. L'Etre et le NEant. Essai d'ontologie phenomenologique. Paris: Editions Gallimard, 1943, p. 492.

${ }^{6}$ Martin Heidegger. Lettre sur l'humanisme. Texte allemand et presenté par Roger Munier. Paris: Editions Aubier Montaigne, 1983. Collection Philosophie de L'Esprit, bilingue, pp. 12-13. La Lettre sur l'Humanisme fut d'abord adressee, en décembre 1946, à Jean Beaufret, en réponse à une lettre en date du 10 novembre. Le nombre pair mis entre parentheses se refere au texte allemand.

${ }^{7}$ Idem. ibidem, p. 125. 
quotidienne. Heidegger avertit ses lecteurs que ce qu'il dit dans Sein und Zeit à propos du 'on' n'a nullement pour objet d'apporter seulement au passage une contribution à la sociologie; pas davantage le 'on' ne désigne-t-il uniquement la réplique, sur le plan moral-existentiel, à l'être-soi ( Selbstsein) de la personne. Ce qui est dit du 'on' contient bien plutôt, sur l'appartenance originelle du mot à l'être, une indication pensée à partir de la question portant sur la vérité de l'être dont le langage est la maison. ${ }^{8}$ Chez Heidegger, le 'on' désigne l'inauthenticité de l'existence quotidienne. La préoccupation de ce que l'on a entrepris avec, pour, ou contre les autres, estime Heidegger, fait que l'on s'inspire constamment du souci de se distinguer d'avec ces 'autres' (die Sorge um einen Unterschied gogen die Anderen); soit que l'on s'efforce seulement d'effacer toute différence avec eux; soit que l'être-là se sentant inférieur, cherche dans ses rapports avec eux, à les égaler, soit encore que l'être-là, se plaçant au-dessus des autres, cherche à maintenir ceux-ci en dessous de lui.9 C'est bien cela que Heidegger entend quand il dit qu'existentialement l'être-en-commun existe sous le signe du distancement (AbständigReit). La distance (der Abstand) demeure ainsi objet d'inquietude et de souci pour la coexistence. Le distancement implique que l'être-là se trouve dans son être-en-commun quotidiensous l'emprise (Botmässigheit) d'autrui ${ }^{10}$ Dans ces conditions, l'être-là n'est plus lui-même; il est aliéné, pourrait-on dire, puisque les autres l'ont déchargé de son être de telle sorte que ses possibilités d'être quotidiennes sont à la discrétion d'autrui.

Cet "autrui" dont parle Heidegger n'est cependant pas quelqu'un de bien déterminé (bestimmte), parce qu'il peut être représenté par n'importe qui. Dans son être-avec-autrui, l'être-là est subrepticement dominé par "autrui". Les "autres" dont chacun fait essentiellement partie, sont ceux qui, dans l'existence commune quotidienne, se trouvent être-là (da sind) de prime abord et le plus souvent ( zunächst und zumeist) ${ }^{11}$. Le 'Qui' ( Wer)ou le sujet de la coexistence quotidienne n'est donc, selon Heidegger, ni celui-ci, ni celui-là, ni soi-même, ni quelques-uns, ni même la somme de tous. Le "Qui", c'est le neutre, c'est-d̀-dire le "on" (das Man) ${ }^{12}$. Le "on" est le tyran innommé, sujet neutre et impersonnel qui assume l'être de la coexistence quotidienne. La tyrannie du "on"

${ }^{8}$ M. Heidegger. Lettre sur l'humanisme, p. 41 (40).

${ }^{9}$ SZ, p. 126.

${ }^{10}$ Ibidem.

"Ibid.

12 Ibidem: "Das Wer" ist das Neutrum, das Man." 
s'explique par le fait que la vie quotidienne me fait ployer sous sa dictature et rien n'échappe à son emprise: préccupations, plaisirs, sentiments, etc. Que de fois nous ne nous amusons pas comme l'on s'amuse? Que de fois ne jugeons-nous pas de la littérature ou de l'art comme l'on juge? Et que de fois ne trouvons-nous pas scandaleux ce que l'on trouve scandaleux? Il est bien évident que le "on" prescrit à la réalité de tous les jours son mode d'être de telle sorte que toute tentative d'originalité est sapée à la base. Autrement dit, le "on" est celui qui "me dicte en tout moment et en toute occasion ce que j'aurai à faire ou a être ${ }^{n 13}$. S'il en est ainsi, quelles sont donc les modalités du "on"?

\section{B. Les modes d'etre du "On"}

Tout être existe d'une certaine façon. Les modes d'être du "on" sont le distancement ${ }^{14}$, la moyenne et le nivellement. La moyenne (die Durchschnittlichkeit) est un caractère existentiel du "on". Le "on" heideggerien demeure toujours dans la moyenne de ce qui est convenable, de ce qui est reçu et de ce qui ne l'est pas, de ce qui mérite l'assentiment et de ce qui ne le mérite pas. ${ }^{15}$ Le souci de la moyenne recele une nouvelle tendance de l'être-là, à savoir le nivellement( die EineDnung) de toutes les possibilités d'être. ${ }^{16}$ Le nivellement universel est à tout propos et avec acharnement. Il s'ensuit que toute nouveauté est considérée comme étant pernicieuse. De même, tout secret et toute personnalité sont combattus sans trêve. Le "on" fait promouvoir l'instauration d'une existence "ouverte" ou d'une opinion publique (die Öffentlichkeit); celle-ci est faite du distancement, de la moyenne et du nivellement. Le public, semble-t-il, a toujours raison. C'est ainsi que l'adage latin dit: Vox populi,vex Dei (la voix du peuple, c'est la voix de Dieu). Bien qu'il intervienne partout avec autorité, le 'on' ou le public est précisément celui qui refuse d'aller au fond des choses ( auf die Sachen); il est aussi celui qui retire à chacun tout sentiment de responsabilité. En d'autres termes, le "on" heideggerien se mêle de tout, mais aussi il se dérobe toujours lorsque l'être-là est acculé à quelques décisions concrètes.

${ }^{13}$ Alphonse de Waelhens. La philosophie de Martin Heidegger. Louvain: Ed. de l'Institut Supérieur de Philosophie, 1942, p. 72 (et 1946, 1948, 1955, 1967) Publications Universitaires de Louvain.

${ }^{14}$ Cfr. supra, note no.9.

15 SZ, p. 127.

${ }^{16}$ Ibidem. 
Il ne court aucun risque à permettre qu'en toute circonstance l'on fasse recours à lui. Il peut aisément assumer n'importe quelle responsabilite, puisqu'à travers lui personne ne peut être interpelle. On dira toujours: "on" l'a voulu. Mais cela pourrait aussi bien signifier que "personne" (keiner) n'a rien voulu. Le "on" ainsi compris est celui qui, dans l'existence quotidienne, décharge (entlastet) l'être-là, le délivre du poids de sa propre existence et satisfait l'instinct de légèreté ( Tendenz zum Leichtnehmen und Leichtmachen) qui dort en tout Dasein. Ce faisant, le "on" nous rend de plus en plus incapables de réaliser nos capacités et nos possibilités propres; et pourtant, il nous accorde également securite, quiétude et assurance. ${ }^{17}$ Dans le royaume du "on" chacun est l'autre et personne n'est soi-même. Le "on", c'est tout le monde et personne. ${ }^{18} \mathrm{~A}$ ce "personne" le Dasein, mêlé à la foule, s'est toujours déjà abandonné. Livré à ce mode d'existence, le Moivéritable ne s'est pas encore retrouvé ou s'est déjà perdu. ${ }^{19}$ Le "on" définit la forme extrême de l'existence en commun ou l'être-avec-autrui. Il est, en dernière analyse, sur le mode de la dépendance et de l'inauthenticite. ${ }^{20}$ Apparemment, ce mode d'être implique un jugement de valeur. Voyons donc, ce que Heidegger en pense.

\section{Ia quiddite do "on" et la morale}

D'aucuns pourraient voir dans la dépendance et l'inauthenticité une perte ou une diminution de la facticité de lêtre-la. Mais Heidegger réfute une telle interprétation. En effet, en se répandant dans le Man, le Dasein ne subit pas une diminution de sa réalité, c'est-à-dire ne devient pas "moins existant".21 En outre, l'être "persone" du Man ne signifie pas que ce dernier est un néant. Man est plutôt une détermination parfaitement positive du Dasein. ${ }^{22}$ L'asservissement du Man, écrit De Waelhens, ne pousse pas le Dasein vers un "être extérieur à soi", mais vers un certain mode de soi qui consiste à ne pas être soi-même. ${ }^{23}$ C'est pourquoi le Dasein existant comme Man-selbst, s'il est un néant de personnalité,

\footnotetext{
${ }^{17}$ Ibid., pp. 127sq; 177 et 268 . De Waelhens, op. cil, p. 73.

${ }^{18}$ Ibid., pp. 128 et 253.

${ }^{19}$ Ibid., p. 128.

${ }^{20}$ Ibidem: "Man ist in der Weise der Unselbständigkeit und Uneigentlichkeit."

${ }^{21}$ De Waelhens, op. cit, p. 64.

2 SZ, p. 129.

${ }^{23}$ Cf. La philosophie de $M$. Heidegger, p. 74.
} 
n'est aucunement un néant pur, mais un "ens realissimum"24. Le Manselbst est le mode selon lequel le Dasein existe le plus souvent. Heidegger nous met en garde contre toute tendance à confondre le Man avec une entité pareille au sujet collectif (allgemeines Subjokt)des sociologues. Le Man est plutôt un existential. Il est aussi peu un étant subsistant que l'être-là lui-même. Heidegger le considère comme "le sujet le plus réel de l'existence quotidienne" ${ }^{n}$. Le Man ne peut être compris comme une sorte de sujet général flottant au dessus d'une pluralité d'individus. Il n'est non plus une propriété constante (bleibende Beschaffenheit) de l'être-là 26 .

Le caractère positif du Man ou du Dasein comme Man-selbst, estime Heidegger, exclut toute appréciation morale négative (negative Wertung) à son sujet ${ }^{27}$. Mais Gurvitch et certains commentateurs pensent que les termes employés par Heidegger pour décrire ce niveau d'existence impliquent d'ores et déjà une appréciation ${ }^{28}$. A ce propos, Joanna Hodge écrit:

The question of ethics is the definitive, if unstated problem of his thinking. ${ }^{29}$

Ce qu'il importe d'éviter, c'est de rattacher la possibilité de l'existence inauthentique à quelque corruption de la nature humaine. En effet, Heidegger ne pose pas et ignore le probleme de la corruption originelle sur lequel la philosophie manque d'arguments décisifs à faire valoir ${ }^{30}$. Ce n'est pas surprenant que l'homme qui a soutenu le nazisme puisse faire semblant d'ignorer le discours moral et moralisant. L'inauthenticité heideggerienne n'est rien d'autre qu'une fuite devant les responsabilités de l'existence personnelle, le refus de s'assumer soi-même. La fuite du Dasein devant lui-même est en dernière analyse, la fuite devant la mort comme finitude radicale dont aucune existence ne peut se passer. ${ }^{31}$ Dans ce sens, l'authenticité ne pourra donc consister qu'à être libre pour la mort.

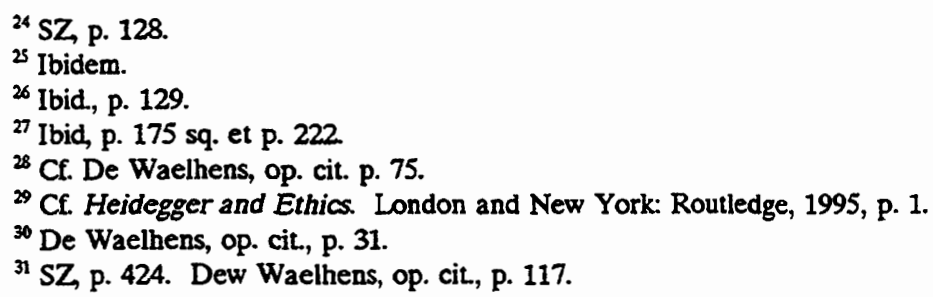




\section{L'AUTHENTICITE OU L'EXISTENCE AUTHENTIQUE}

Si en effet, la tyrannie exercée par le Mans'avère problématique pour l'existence authentique du Dasein, alors une existence qui se veut authentique doit se ressaisir et se soustraire aux tentations et à la dispersion du $\mathrm{Man}^{32}$.Toute authenticité ou toute démarche vers elle présuppose et débute par un Zurückholen, ${ }^{33}$ s'amorce par une conversion. La liberté pour la mort (Freibeit zum Tode) est la condition sine qua non de possibilité et la piece essentielle de l'authenticité heideggerienne.

\section{A. La mort et la conscience morale}

Exister authentiquement, c'est se comprendre comme mourant. Cette compréhension est intimement liée à la déréliction du Dasein jeté dans le monde et son angoisse de la mort. Dans ce contexte précis, l'angoisse n'est pas la crainte de la mort. Elle est plutôt due à la possibilité de l'inauthenticité. La conversion qui marque le passage de l'inauthenticité à l'authenticité se manifeste dans la voie de la conscience (die Stimme des Gewissens). ${ }^{34} \mathrm{La}$ conscience morale appartient à l'existence humaine et $y$ joue un rôle révélateur comme témoignage de l'existence authentique. Elle est un retour sur soi du Dasein aliéné ou perdu dans le Man et dans la quotidienneté (die Alltäglichkeit). Selon le dire de De Waelhens, la conscience morale est:

ce pouvoir d'interpellation radicale qui s'adresse à nous lorsque nous sommes perdus dans les divertissements mondains. ${ }^{35}$

L'interpellation est un appel silencieux de la conscience morale qui détruit l'architecture de l'existence quotidienne. Cet appel est et doit être silencieux parce que selon Heidegger, toute parole est bavardage (Gerede) et en tant que tel elle est mondaine. La mondanité de la parole signifie qu'elle traite "de quelque chose" et nourrit par là les tendances de la préoccupation. Bien qu'elle soit muette, l'interpellation est un cri qui donne à penser ${ }^{36}$. Comme la conscience chez E. Husserl, l'interpel-

\footnotetext{
${ }^{32}$ De Waelhens, op. cit., p. 31.

${ }^{33} \mathrm{SZ}$, p. 268 . Ce mot allemand signifie aller rechercher, reprendre.

${ }^{34}$ Ibidem.

${ }^{35}$ lbid, p. 153.

${ }^{36}$ Ibid., p. 271: "... Stimme ist aufgedasst als das Zu-verstehen-geben."
} 
lation est intentionnelle, c'est-à-dire elle présuppose un sujet qui interpelle, un objet interpelle et un message à communiquer. Heidegger identifie l'appelant, l'appele et le contenu ou la signification de l'appel en faisant l'ontologie de la conscience morale.

\section{B. Ontologie de la conscience morale}

En vue de faire lontologie de la conscience morale, Heidegger pose et répond à trois questions, à savoir: (1) Qui appelle? (Wer rutt?) (2) Qui est appelé ou interpellé? et (3) Que signifie cet appel (interpellation/ avertissement)?

L'appelant ou l'interpellateur (der Ruter) est le Dasein à la fois mien et étranger. Il est comparable à une force etrangère (frewden Macht) à nous-mêmes qui s'élève en nous et s'adresse à nous, et cela malgré nous. L'extériorité apparente de l'appelant a fait que d'aucuns l'ont identifié avec Dieu (Gott), soit avec des réminiscences en des forces sociologiques, voire biologiques. Mais l'appelant est, en dernière analyse, le Dasein en état de détresse et de nuđité de sa condition originelle ${ }^{37}$. En d'autres mots, commente De Waelhens, l'appelant n'a pas d'état civil. Il n'est ni un conseil de la société, ni l'image de nos parents surgissant en nous pour nous porter un secours pendant un moment difficile, ni le souvenir de tel ami, ni le sentiment de l'honneur ou du devoir... L'appelant est indéterminable, car la conscience est toute dans son cri.

L'interpelé (der Angerutene) est de nouveau le Dasein pris par le monde et enchaîné, aliéné et perdu ou dispersé dans le Man. L'appel ou l'interpellation (der Ruf) est une invitation à me reconnâtre coupable (schuldig). C'est le cri ou l'avertissement du souci ${ }^{38}$. L'appel signifie le suprême effort de ce qui va être refoule, mais ne veut pas l'être, parce qu'il définit la suprême et l'unique verité. En égard à la signification de l'interpellation, il est évident, comme De Waelhens l'a bien vu et dit, que l'existence humaine est un oubli dans une grande part. Elle est toujours conquête et jamais une possession ou un acquis. Si donc, chez Heidegger, l'interpelant et l'interpellé sont le même Dasein, quelles seraient alors les relations de l'un à l'autre? A cette question, il répond:

L'interpellant est le Dasein qui, dans sa déréliction, s'angoisse pour ce qu'il pourra être. L'interpellé est ce méme Dasein que la conscience Dasein.

${ }^{37}$ SZ pp. 275 sq. La condition originelle dont Heidegger parle, c'est la déréliction du

${ }^{38}$ Ibidem, pp. 274, 277, 286, 289 et 317. 
morale veut restituer à son pouvoir-etre authentique en le retirant, par son appel, de sa chute dans le $\mathbf{M a n}^{39}$.

La dialectique de la conscience morale se trouve rattachee en chacun de ses éléments à l'un des caracteres du souci. Celui auquel la conscience en appelle est le Dasein en tant que porteur de l'option fondamentale entre l'authenticité et l'inauthenticité. Bref, l'appel vient de moi et s'adresse à moi-même au-delà de moi (aus mir, an mich, über mich) ${ }^{\text {to }}$. L'identification de la conscience morale à des forces étrangères à nous-mêmes n'est pas plausible. En effet, un Dieu extérieur est le piège du Man. En outre, Heidegger est, quoique d'une manière floue, contre l'hypothèse divine. De Waelhens explique la position de Heidegger en estimant que le fait de faire appel a un autre être est une manoeuvre de diversion destinée à cacher la véritable révélation apportée à la conscience morale ${ }^{41}$. Il est a noter cependant que les interpretations de la conscience morale chez Heidegger ne sont pas du tout naturelles, puisque normalement la conscience avertit, conseille et reproche; elle n'est ni insinuante; ni péremptoire; elle est moins vague que plus concrète. Elle s'exprime en relation avec des faits precis qu'elle interdit ou commande, puisqu'elle blâme ou approuve; et puis la conscience est surtout critique. Elle joue, pour employer les mots de De Waelhens, "le rôle d'un tribunal chargé d'apprecier les actions qui remplissent notre vie" ${ }^{42}$. Heidegger n'en disconvient pas. Et pourtant, la réponse que son Dasein doit donner à l'appel de la conscience est spéciale, à savoir linstauration soit de l'authenticité, soit de l'inauthenticité. L'authenticité est le but de l'interpellation. Car, explique De Waelhens, l'appel se propose de rendre le Dasein à l'exercice de ses possibilités authentiques, c'est-à-dire à amener le Dasein au refus de l'existence dispersée. Il ne s'agit surtout pas de conseiller telle ou telle autre possibilité concrète et déterminée, puisque l'authenticité ne consiste pas à faire ceci plutôt que cela, mais à faire ceci ou cela d'une certaine maniere $e^{43}$. Le refus de l'inauthenticite n'est possible chez Heidegger que grâce à un acte laîc de mea culpa, c'est-à-dire l'aveu de culpabilité.

${ }^{39}$ SZ, p. 277. Cite par De Waelhens, op. cit., p. 158.

* Heidegger, cite par De Waelhens, ibid.

De Waelhens, op. cit. p. 158.

2 Ibid.

${ }^{13}$ Ibid. p. 159. Le passage de l'inauthenticité à l'authenticité s'avère difficile, car la dictature du Man est omniprésente. 


\section{Conscience morale et culpabilite}

L'existence authentique à laquelle appelle la conscience morale est intimement liée à la culpabilité (Schuldigeein). L'interpellation de la conscience morale est une invitation à nous reconnaître coupables ${ }^{44}$. La conscience morale invite le Dasein à accepter sa cupabilité fondamentale. Celle-ci se trouve dans la négativité, c'est-à-dire la mort. Il faut noter que le mot allemand Schuld signifie aussi bien 'faute' que 'dette'. La notion de 'faute' est liée à l'idée de 'responsabilité' et à celle de 'cause'. Chez Heidegger l'idée de 'faute' énonce que le Dasein coupable est la source ou la cause d'un manque dans ses propres biens. Ma culpabilité consiste en ce qu’à cause de moi-même, je suis privé de quelque chose qui m'appartient de iure. Pour le Dasein, la culpabilité signifie être source ou fondement d'une négativité (Grundsein einer Nichtigkeit)'s. Le Dasein peut fonder une négativité chez quelqu'un d'autre, parce qu'il est lui-même affecté de négativité. En outre, lorsque le Dasein commet une faute ou porte atteinte extérieure au droit ou à la moralité, ce n'est pas cette lésion comme fait qui le culpabilise; mais la perpétration de ce fait n'est elle-même possible que parce qu'il y a déjà en moi une source ou un fondement de négativité qui est le mal véritable ${ }^{46}$. La culpabilité originelle du Dasein, celle qui engendre les autres fautes concrètes qu'il commet, consiste en ce qu'il est fondement de négativité. Celle-ci réside dans le fait que le Dasein ne pourra jamais devenir maître absolu de son existence. Cette existence souffre d'une impuissance, c'est-z̀-dire un néant ou une finitude originelle et sans remède. La négativité est omniprésente, elle assaille le Dasein tant dans son commencement que dans sa fin; elle est inhérente au Dasein en tant que souci (Sorge) ${ }^{47}$.

Du moment que le Dasein accepte d'assumer sa déréliction (Geworfenheit) et entreprend de se faire, il consent à mener une existence imparfaite et c'est là précisément que résident le péché et la faute. L'acceptation de la finitude rend le Dasein 'coupable' et 'fautif. Chez Heidegger l'idée de 'péche' est dépourvue de toute référence théologique. Autrement dit, le péché n'a rien à voir avec l'amour de Dieu et du prochain qui constitue la synthèse du Décalogue. C'est pourquoi J.

4 SZ, p. 280.

45 Ibid., Pp. 283 et 305.

4 Ibid, p. 284: "...diese wird erst möglivh 'auf Grund' eines ursprünglichen Schuldigseins." Cette phrase met en évidence ce que je considère comme déterminisme heideggerien.

7 Ibid., p. 285. 
Wahl dit que la philosophie de Heidegger se présente comme une laïcisation de la pensée de $S$. Kierkegaard ${ }^{48}$. A ce même propos, E. Lévinas écrit, quant à lui, que la pensée de Heidegger s'annonce comme "un discours sur 1 'absence de Dieu"49.

Le Dasein existe donc authentiquement lorsque, repondant à l'appel qu'il fait à lui-même, et lorsque, reconnaissant et assumant sa culpabilité, il se trouve ainsi rejete vers ses possibilités personnelles d'existence $^{50}$. Si l'existence authentique ou résolue (Entschlossenheit) consiste à assumer dans le silence le plus absolu et dans l'angoisse la culpabilité fondamentale qui comprend par avance ou par anticipation toutes les possibilités du Dasein ${ }^{51}$. Elle est intimement liée à l'être-pour-la-mort (Sein zum Tode); elle est la fidélité de l'existence à elle-même; elle consiste à "voir toutes choses et à tout instant sub specie mortis"s2. La mort est le phénomène en quoi le Dasein se réhabilite, c'est-à-dire devient proprement lui-même (eigentlich selbst) ${ }^{53}$. La mortalité est une propriété propre à chacun, une propriété privée dans laquelle chacun joue son être dans sa totalite. Par consequent, chacun doit mourir de sa propre mort. Personne ne peut mourir à la place d'un autre $^{54}$. Le Dasein existant authentiquement vit dans l'attente, dans la présence perpétuelle et dans l'incessante anticipation de la mort (Vorlaulen des Todes). Mais l'authenticité est une conquête toujours précaire, car l'inauthenticité est une possibilité structurelle du Dasein,et puisque la dictature du Man est partout.

\section{L'existence resolue et le Man}

Etant un retour sur soi ou un processus de désaliénation, l'authenticité ou l'existence résolue ne provoque pas pour autant une attitude de splendide isolement, puisqu'elle est édifiée sur la nécessité pour l'homme d'être-dans-le-monde et d'être-en-commun ${ }^{55}$. Autrement dit, explique De

46 Jean Wahl, "Etudes kierkegaardiennes", in Bulletin de la Societe trançaise de philosophie, p. 26.

49manuël Levinas cite par de Waelhens, op. cit., p. 164.

${ }^{50}$ De Waelhens, op. cit., p. 169.

si SZ p. 297. Langoisse est le sentiment lie à la perception de la solitude du Dasein en quête de l'authencité.

${ }^{52}$ Cité par de Waelhens, op. cit., p. 176.

${ }^{53} \mathrm{SZ}$, p. 322

\$4 Idem, p. 240: "Keiner kann dem Anderen seing Sterben abnehmen."

ss Ibid., p. 298. 
Waelhens, "l'existence résolue seule nous fait accéder à l'être-encommun", tandis que l'existence inauthentique ne connaissait que d'equivoques (Zweideutigkeiten)et de tyranniques associations d'intérêts et de préoccupations ${ }^{56}$. L'existence résolue nous délivre de l'obsession et de la tyrannie de nos préoccupations en nous faisant considérer chaque chose sub specie mortis, à la lumière de l'hyper-puissance (Ubermacht) $)^{57}$. L'existence résolue n'impose pas des contenus d'existence, puisque cela ne releve pas de la compétence de la philosophie existentiale de déterminer ce qu'il faut exécuter concrètement dans l'existence authentique. La philosophie existentiale ne s'interesse pas du tout à l'éthique normative proprement cité dont la tâche principale est de prescrire des préceptes moraux à observer. Heidegger ne traite pas des préceptes, car, selon lui:

L'obligation morale apparaît comme antithese de l'être dès que celujci se definit comme idée $e^{58}$.

En outre, l'infinité des situations concrètes constamment modifiées ou modifiables et le fait de la liberte justifient l'attitude de la philosophie existentiale vis -à-vis de l'éthique traditionnelle. Finalement, Heidegger fait sienne l'opinion de Kierkegaard selon laquelle la philosophie doit réserver à l'existence "die offene bleibende Entschiedenheir","s9 c'est-dे-dire la résolution qui reste ouverte (littéralement). En tant que catégorie philosophique, ou mieux, en tant qu'existential universellement, concrètement et absolument valable, l'Entschlowsenheit doit rester pleinement indéterminée quant à son contenu. En effet, ce n'est point en faisant ceci ou cela que nous existons authentiquement, mais c'est plutôt en le faisant d'une certaine manière et dans une certaine perspective.

Bien qu'il soit difficile d'échapper à la tyrannie du Man, l'existant résolu est le seul à avoir conscience de la situation comme telle, c'est-à-dire la dictature du Man. Par contre, l'existant journalier ou inauthentique ignore cette situation à tel point qu'il se représente l'existence comme un mélange incohérent de hasards (Zufälle) et d'actes

So Cf. La philosophie de Martin Heidegger, p. 172

${ }^{57} \mathrm{SZ}$, p. 384. Cite par De Waelhens, op. cit., p. 172

${ }^{58}$ Cf. Einführung in die Metaphysik. Tübingen: Niemeyer, 1953, 1958, 1967, 1976, p. 150. "Das Sollen tritt als Gegensatz zum Sein auf, sobald dieses sich als Idee bestimmt."

${ }^{99}$ Soren Kierkegaard. Vernunft und Existenz, p. 48. Voir aussi Kierkegaard. Oeurres complètes. Paris: Ed. Gottsched et Schrempf. Ed. de l'Orante, 1966. 
libres. En d'autres mots, l'existence quotidienne n'accède pas à la situation. Ne connaissant que la situation générale, l'existence inauthentique vit dans l'absurde deroulement des circonstances (Gelegenheiten), puisqu'elle est incapable de réaliser la synthèse de l'impose et du voulu. Vivre une situation est donc l'apanage de la resolution authentique, car l'existence inauthentique ne fait que subir les situations ${ }^{60}$. En dépit de son manque d'intérêt à l'éthique traditionnelle, Heidegger, comme tout grand philosophe, n'a pu s'empêcher de contribuer au discours sur l'éthique. Sa théorie n'est ni normative, ni méta -éthique, mais originale. Elle s'appelle éthique originelle.

\section{L'ETHIQUE ORIGINELLE}

\section{A. Heidegger et Tethos}

Peut-on vraiment parler d'une éthique chez Heidegger, etant donné qu'il n'a jamais écrit de traités d'éthique? En effet, bien qu'il ne se soit jamais proposé de faire de la morale, Heidegger a explicitement dénoncé l'éthique traditionnelle en tant qu'elle est intimement liée au nihilisme ${ }^{61}$. Il est donc possible de parler d'un ethos dans le sens d'une éthique originelle (ursprüngliche Ethik) comme séjour de l'homme dans la vérité de l'Etre. Pour examiner le problème éthique chez Heidegger, force nous est de recourir a la Lettre sur l'humanisme. Dans cette oeuvre, Heidegger se rapporte à trois questions posées par Jean Beaufret, à savoir: (1) "Comment peut-on donner un sens au mot 'humanisme'?"62 (2) "Ce que je cherche à faire, depuis longtemps déjà, c'est préciser le rapport d'une ontologie avec une éthique possiblen ${ }^{n}$.(3) "Comment sauver l'elément d'aventure que comporte toute recherche sans faire de

\footnotetext{
${ }^{60} \mathrm{SZ}$, p.300. Le concept heideggerien de "situation" est assez semblable à celui de Karl Jaspers. Mais chez Heidegger, "situation" se dit seulement de l'authenticite; il ne distingue pas explicitement, comme le fait Jaspers dans sa Psychologie der Weltanschauungen (1919, 1925, 1932), entre "situation" tout court et "situation-limite" (Grenz-Situation). Voir aussi Gabriel Marcel, "Aperçu phenomenologique de l'être en situation," in Recherches philosophiques, 1936-1937, article repris dans son livre intitule Du refus à l'invocation, Paris, 1942, pp. 111-138.

${ }^{61} \mathrm{C}$. A Lalande, op. cit. Lalande definit le nihilism comme "la doctrine selon laquelle il n'y a point de verite morale, pas de hitrarchie des valeurs. Le nihilisme se pose la question "A quoi bon?" et n'y peut répondre.

Q M. Heidegger, Lettre sur l'Humanisme, pp. 33, 115.

Ibidem, p. 139.
} 
la philosophie une simple aventurière"? ${ }^{64}$ De ces trois questions, seule la deuxième nous intéresse. L'histoire de la philosophie nous apprend qu'aucun grand philosophe digne de ce nom ne s'est passé de l'éthique. Ainsi par exemple, Socrate a fait de la science morale la pierre angulaire de sa philosophie. Aristote n'a pu s'empêcher, quant à lui, d'écrire I'Ethique à Nicomaque. Kant s'est immortalisé non seulement grâce à sa révolution copernicienne, mais aussi à son imperatif catégorique. Mais qu'en est-il de Heidegger? Peu après la publication de son chef-d'oeuvre L'Etre et le Temps, un jeune ami demanda Heidegger: "Quand écrivezvous une éthique?" ${ }^{65}$ De la réponse que Heidegger donna à cette question, il est clair qu'il reconnaissait l'importance de l'éthique dans la société moderne dominée par la technique. Plus précisément Heidegger déclara:

\begin{abstract}
"Là où l 'essence de l'homme est pensee de façon aussi essentielle, c'est-à-dire à partir uniquement de la question portant sur la vérité de l'Etre, mais où pourtant l'homme n'est pas érige comme le centre de l'étant, il faut que s'eveille l'exigence d'une intimation qui lie, et des règles disant comment l'homme, experimente à partir de l'ek-sistence de l'Etre, doit vivre conformement à son destin. Le voeu d'une éthique appelle d'autant plus impérieusement sa réalisation que le desarroi evident de l'homme, non moins que son désarroi cache, s'accroissent au-delà de toute mesure. A cet établissement du lien éthique, nous devons donner tous nos soins, en un temps où il n'est possible à l'homme de la technique, voue a l'etre collectif, d'atteindre encore a une stabilite assuree, qu'en regroupant et ordonnant l'ensemble de ses plans et son agir conformement à cette technique ${ }^{66}$.
\end{abstract}

Au fond, il n'est possible de parler d'une éthique au sens originel chez Heidegger, que sur la base de l'humanitas au service de la vérité de l'Etre, c'est-à-dire l'humanité comme ouverture ek-statique de l'homme à la vérité de l'Etre. Autrement dit, on peut identifier une dimension éthique dans la pensée heideggerienne, à condition que l'on remonte à l'horizon ontologique dans lequel l'homme est considéré comme ouverture essentielle à l'Etre ${ }^{67}$.

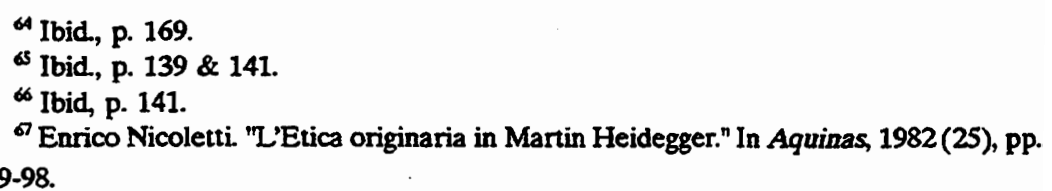




\section{B. Ethique et Ontologie}

\section{Qu'est-ce que l'ethique?}

Heidegger nous rappelle que l'éthique apparaît pour la première fois avec la logique et la physique dans l'Ecole de Platon. Ces dernières disciplines prennent naissance à l'époque où la pensée se fait philosophie, et la philosophie, epistemé (science, Wissenschaft), et la science elle-même, affaire d'école et d'exercice scolaire ${ }^{68}$. Heidegger croit que l'essence de l'ethos s'éclaire par cette sentence d'Héraclite: "Ethos anthropo daimon. ${ }^{n 69}$. Cette sentence se traduit d'ordinaire comme suit: "Le caractère propre d'un homme est son démon". Ethos signifie séjour ou Autenthalt en allemand, lieu d'habitation. Il désigne la région ouverte où l'homme habite ${ }^{70}$. La sentence d'Heraclite veut dire que l'homme habite, pour autant qu'il est homme, dans la proximité du Dieu (daimon) $^{71}$. En d'autres mots, explique Héraclite:

Le sejour (accoutume) est pour l'homme le domaine ouvert à la presence du Dieu (de l'insolite). ${ }^{72}$

Conformément au sens fondamental du mot ethos, le terme 'éthique' indique que cette discipline se réfere au séjour de l'homme. L'éthique dont il est question chez Heidegger est une éthique originelle (ursprüngliche Ethik), c'est-à-dire celle qui pense la vérité de l'Etre comme l'elément originel de l'homme en tant qu'ek-sistant. Une telle éthique n'a donc encore rien à voir avec la science ayant pour objet le jugement de valeur en tant qu'il s'applique à la distinction du bien et du mal ${ }^{73}$.

${ }^{68}$ Lettre sur l'Humanisme, p. 143.

${ }^{69}$ Fragment 119, cité par Heidegger dans ta Lettre, p. 145.

${ }^{70}$ Lettre, p. 145.

$n$ Ibid, p. 145.

$\pi$ Cité par Heidegger, ibid, p. 151: "Der (geheure) Aufenthalt ist dem Menschen das offene für die Anwesung des Gottes (des Ungeheuren)", ibid., p. 150.

${ }^{73}$ Notons en passant que les philosophes speculaltifs allemands qui suivent Kant font une distinction entre "Ethique" et "Morale" et mettent la première au-dessus de la seconde. Ainsi par exemple, Schelling dit que la morale en general pose un commandement qui ne s'adresse qu'a l'individu, et n'exige que l'absolue personalité (Selbstheit) de l'individu; tandis que l'ethique pose un commandement qui suppose une societé d'êtres moraux et qui assure la personnalité de tous les individus par ce qu'elle exige de chacun d'eux. Cf. Oeurres, I, 252. Pour Hegel, par contre, "Morale designe plutôt le domaine de lintention subjective; "Ethique", le regne de la moralite (Situlichkeit). Cf. Lalande , op. cit, observations 


\section{Qu'est-ce que Pontologie ou la metaphysique?}

C'est la science de l'Etre en tant qu'être et en tant que cause première de tout étant. Ethique et ontologie sont intimement liées. Bien qu'il reconnaisse leur relation, Heidegger n'explique pas en termes clairs en quoi elle consiste. Au contraire, il se contente d'échappatoires lorsqu'il dit que c'est une question sans fondement, parce que:

La pensé qui pose la question de la verité de l'Etre, et par la même détermine le séjour essentiel de l'homme à partir de l'Etre et vers lui, n'est ni éthique, ni ontologie. ${ }^{74}$

Il ajoute ensuite qu'une ontologie doit être completée par une éthique. Il remonte à l'expérience éthique qui envahit les tragedies de Sophocle. Celles-ci, estime Heidegger, "abritent plus originellementl' ethos dans leur dire, que les leçons d'Aristote sur $l^{m}$ Ethique ${ }^{n} .{ }^{75}$ En outre, le Fragment 119 d'Héraclite cité plus haut est un bel exemple d'une éthique originelle. Celle-ci n'est ni une détermination de valeurs, ni une fixation de normes et d'obligations, mais elle est la pensée même de l'Etre. Heidegger conclut:

Si donc, conformement au sens fondamental du mot 'ethos', le terme d'ethique doit indiquer que cette discipline pense le sejour de l'homme, on peut dire que cette pensee qui pense la verite de l'Etre comme l'élément originel de l'homme en tant qu"eksistant" est déjà en elie-mème l'ethique originelle ${ }^{\prime 6}$.

Enrico Nicoletti clarifie le concept d'éthique originelle en disant qu'elle n'est rien d'autre que la pensée de l'Etre. Elle n'a rien à voir avec la discipline qui trouve son fondement soit dans une ontologie, soit dans une philosophie transcendantale. Par ailleurs, l'ethique entendue comme discipline renvoie à une ontologie et partant, à la métaphysique comme science de l'Etre en tant qu'être; tandis que l'éthique originelle surgit seulement dans le cadre de la vérité de l'Etre comme différence, c'està-dire comme l'élément originel de l'homme; elle est la racine occulte tant de l'éthique que de l'ontologie comme discipline ${ }^{\pi}$.

\footnotetext{
historiques sur le mot "Ethique."

${ }^{74}$ Lettre sur l'Humanisme, p. 154 sq (252).

75 Ibid., p. 145.

76 Ibid., p. 145.

${ }^{7}$ Cf. "L'Etica Originaria in M. Heidegger," p. 448.
} 


\section{Conclusion}

Dans sa conception du "public", Kierkegaard disait que chaque époque historique est caractérisée par sa propre dépravation. Dans les systemes dits communautaristes, avant l'avenement de la révolution industrielle, du capitalisme et de l'individualisme, et même pendant la première moitie du XXè siècle, le mépris quasi total de l'individu était une des dépravations majeures. Il serait tout à fait juste de dire, en paraphrasant Kierkegaard, que l'inauthenticité dont parle Heidegger est l'existence dans une époque dont le trait caractéristique est le mépris de l'individu et tout ce qui est individuel. Dans une telle epoque, tout fait partie d'un mouvement de masse, par exemple le National-Socialisme en Allemagne hitlérienne, le Fascisme mussolinien en Italie, le Communisme en Union Soviétique, etc. Dans l'existence inauthentique, les membres du mouvement s'y perdent, ou bien ils se perdent dans la totalite des choses et de l'histoire universelle. Ils troquent leur identite personnelle contre l'identité collective. Tous ceux et celles qui s'enfoncent dans le mouvement, l'universel ou le public - fût-il l'Etat, le parti-Etat ou l'Esprit Absolu à la Hegel - ont tendance à rejeter toute responsabilite personnelle ${ }^{78}$.

Contrairement à ce que pense Heidegger, le "on" est non seulement le sujet le plus réel de l'existence quotidienne, mais aussi le public, le mouvement de masse, conditionnement socio-culturel, la pression exercee par nos égaux (peer pressure), et la socialisation excessive au détriment des libertés, des droits et de la croissance des individus. C'est le mépris de l'individu. D'aucuns ont pensé, à tort ou à raison, que l'individualisme et le solipsisme sont une révolte contre le conditionnement social. A ce propos, par exemple, David Reisman écrit:

Social science has helped us become more aware of the extent to which individuals, great and little, are the creatures of their cultural conditioning; and so we neither blame the little nor exalt the great. But the same wisdom has sometimes led us to the fallacy that, since all men have their being in culture and as the result of the culture, they owe a debt to that culture which even a lifetime of altruism could

\footnotetext{
${ }^{78}$ Par exemple, en automne 1995, une émeute de trois jours eut lieu à Livingston, en Zambie. Elle était due aux tensions raciales et soci-economiques entre la population noire et celle d'origine asiatique. Les émeutes saccagèrent les magazins et les usines, détruisirent des voitures Mercedes et pillerent maisons et temples hindous. A la question de savoir qui ont causé ces dégâts, les citadins répondirent: "Tout le monde." (Cfr. The Baltimore Sun, Friday, January 26, 1996, p. 2A).
} 
not repay. Sometimes the point is pushed to the virtual denial of individuality: since we arise in society, it is assumed with a ferocious determinism that we can never transcend it. All such concepts are useful correctives of an earlier solipsism. But if they are extended to hold that conformity with society is not only a necessity but also a duty, they destroy that margin of freedom which gives life its savor and its endiess possibility of advance 7 .

Le reniement de l'individualité, le conformisme et le déterminisme sont évidents chez Heidegger, et plus précisément quand il dit par exemple que ce n'est pas moi, au sens du moi-même, qui vis, mais c'est plutôt les autres, dont le mode d'être est celui du "on", qui vivent; ou que le Dasein est le "on" et demeure tel le plus souvent. Il n'y a donc point de doute que ces déclarations ont des répercussions non moins désastreuses et inquiétantessur la responsabilité personnelle. Ma responsabilité est celle de tout le monde; mes actes et mon être-même sont ceux des autres et échappent à mon contrôle. Mais si ce n'est jamais moi qui suis ou agis, mais les autres qui sont et agissent en moi, ou pire encore, si le Dasein peut fonder une négativité chez quelqu'un d'autre, parce qu'il est lui-même affecté de négativité, alors quelle conclusion pourrait-on tirer du soutien de Heidegger au Nazisme? Peut-il avoir mené des actions nazies en tant que lui-même, Monsieur Heidegger ou "le Heidegger ontique" ? Ou plutôt les a-t-il subies ou menées en tant que "le Heidegger ontologique", c'est-à-dire "le Dasein-Heidegger", dont l'identité est monsieur-tout-le-monde-et-personne?Cette question ouvre la porte à un véritable débat moral que je ne voudrais pas entamer ici. Qu'il me suffise cependant de dire que les concepts d'authenticite et d'ethique originelle voilent, bon gré mal gré, les intentions et les actions nazies du "Camarade" Heidegger. La liberté pour la mort - qui est la condition sine qua non de possibilite et la piece essentielle de l'existence authentique n'est-elle pas liée à "la dernière solution" ou l'holocauste? Autrement dit, le rôle joue par Heidegger dans le National-Socialisme porte à croire que sa pensée philosophique renferme la conviction du Nazisme suivant laquelle les Juifs "méritaient" la mort et devaient l'accepter comme leur "possibilité authentique". Mais qu'est-ce que la liberté pour la mort pour des milliers de Juifs qui ont eté forcés à mourir dans les chambres à gaz d'Auschwitz? La mort ne represente-t-elle pas une situation-limite pour le moribond, c'est-à-dire la néantisation de toutes ses possibilités, et pourtant, n'en fait plus partie? Et qu'est-ce que la liberté pour la mort

${ }^{79}$ Cfr. Individualism Reconsidered. New York: Doubleday, 1954. 
pour d'autres millions de gens qui, à cause de flagrantes violations des droits de la personne par des régimes totalitaristes, ne meurent pas de leur belle mort? En collaborant avec le Nazisme, Heidegger était coupable de fonder une négativité chez les victimes de l'holocauste. Au fond, chez lui, authenticité et éthique (conscience morale) prêtent le flanc à une veritable thanatologie. En fait elles sont une thanatologie camouflée. 